\title{
Evaluation of internal transcribed spacer 2-RFLP analysis for the identification of dermatophytes
}

\author{
Correspondence \\ Mario Vaneechoutte \\ Mario.Vaneechoutte@UGent.be
}

Received 29 June 2009

Accepted 18 September 2009

\author{
Thierry De Baere, ${ }^{1} \dagger$ Richard Summerbell, ${ }^{2} \ddagger$ Bart Theelen, ${ }^{2}$ \\ Teun Boekhout ${ }^{2,3}$ and Mario Vaneechoutte ${ }^{1}$
}

\author{
${ }^{1}$ Laboratory for Bacteriology Research, University of Ghent, B-9000 Ghent, Belgium \\ ${ }^{2}$ CBS Fungal Diversity Centre, Uppsalalaan 8, 3584 CT Utrecht, The Netherlands \\ ${ }^{3}$ Division of Acute Medicine and Infectious Diseases, University Medical Centre Utrecht, Utrecht, \\ The Netherlands
}

\begin{abstract}
A total of 95 isolates, belonging to 33 species of five dermatophyte genera, i.e. Arthroderma (15 species), Chrysosporium (two), Epidermophyton (one), Microsporum (three) and Trichophyton (12), were studied using internal transcribed spacer 2 (ITS2)-PCR-RFLP analysis (ITS2-RFLP), consisting of amplification of the ITS2 region, restriction digestion with BstUI (CG/CG) and restriction fragment length determination by capillary electrophoresis. ITS2-RFLP analysis proved to be most useful for identification of species of the genera Arthroderma, Chrysosporium and Epidermophyton, but could not distinguish between several Trichophyton species. The identification results are in agreement with established and recent taxonomical insights into the dermatophytes; for example, highly related species also had closely related and sometimes difficult-to-discriminate ITS2-RFLP patterns. In some cases, several ITS2-RFLP groups could be distinguished within species, again mostly in agreement with the taxonomic delineations of subspecies and/or genomovars, confirming the relevance of ITS2-RFLP analysis as an identification technique and as a useful taxonomic approach.
\end{abstract}

\section{INTRODUCTION}

The dermatophytes are a group of closely related fungi with the capacity to invade keratinized tissue, mainly of mammals, causing a cutaneous infection generally restricted to the non-living cornified layers, commonly referred to as 'ringworm'. The genera Epidermophyton, Microsporum and Trichophyton comprise anamorph (asexual or imperfect) species, of which some, mostly the zoophilic and geophilic species of the genera Microsporum and Trichophyton, can reproduce sexually, producing ascomata with asci and ascospores; these are classified in the teleomorph genus Arthroderma, family Arthrodermataceae of the Onygenales, phylum Ascomycota. Some Chrysosporium species have teleomorphs in the genus Arthroderma, but are not

tPresent address: Scientific Institute of Public Health, Department of Bacteriology, Juliette Wytsmanstraat 14, 1050 Brussels, Belgium.

‡Present address: Sporometrics Inc., 219 Dufferin Street, Suite 20C, Toronto, ON M6K 1Y9, Canada.

Abbreviation: ITS2, internal transcribed spacer 2.

The GenBank/EMBL/DDBJ accession number for the sequence of the ITS2 region of Chrysosporium carmichaelii CBS 269.77 is FM212440.

ITS2 sequence results are available as supplementary material with the online version of this paper. considered to be true dermatophytes, as this designation should be restricted to infectious organisms.

The diagnosis of ringworm is reached by the detection of hyphae by direct microscopic examination of the clinical sample, and subsequent identification is commonly carried out by a combination of microscopic morphology of spores and hyphae, and macroscopic characteristics such as the morphology and colour of the colonies. Sometimes subculture on different media is required, so final identification can be laborious and time-consuming. In addition, this classical approach relies strongly on the specific expertise of the technicians, especially for uncommon dermatophytes.

During the last decade, a wide variety of molecular techniques has become available as possible alternatives for routine identification of fungi in clinical microbiology laboratories (Arabatzis et al., 2006, 2007; De Baere et al., 2002; Erhard et al., 2008; Turenne et al., 1999).

Ribosomal internal transcribed spacer 2 (ITS2)-PCR is a PCR-based approach using universal fungal primers, followed by fragment analysis of the amplicon by capillary electrophoresis, and has been shown to be useful and reliable for the identification of yeasts (De Baere et al., 2002, 2005; Turenne et al., 1999). ITS2 sequencing has already been applied to different groups of filamentous 
fungi (Gräser et al., 1999; Summerbell et al., 1999). Here, we evaluated the discriminatory power of ITS2-PCR in combination with restriction digestion using BstUI for the identification of dermatophyte fungi.

\section{METHODS}

Strains. All strains used in this study were obtained from the Centraal Bureau voor Schimmelcultures (CBS; Utrecht, The Netherlands). The taxonomic status of most of these strains was established during previous morphological and molecular studies. Table 1 presents the species and strains studied.

DNA extraction. After culture of the strains on Sabouraud agar (Becton Dickinson), a piece of mycelium was transferred to distilled water for transportation.

DNA extraction was carried out using a genomic DNA extraction kit (Qiagen) according to the manufacturer's instructions, preceded by lyticase digestion. Lyticase digestion was carried out by transferring a loopful of mycelium to a $1.5 \mathrm{ml}$ tube containing $2.5 \mathrm{mg}$ zymolase (Sigma-Aldrich) in $250 \mu \mathrm{l} \mathrm{zymolyase} \mathrm{lysis} \mathrm{buffer}[10 \mathrm{mM}$ Tris/HCl ( $\mathrm{pH} 8.0), 1 \mathrm{mM}$ EDTA] and incubating for $45 \mathrm{~min}$ at $37^{\circ} \mathrm{C}$. Subsequently, $200 \mu \mathrm{l}$ was transferred to another tube to carry out the DNA extraction, which resulted in $100 \mu \mathrm{l}$ of DNA extract. The DNA from some strains was isolated using the CTAB method, as described previously (O’Donnell et al., 1997).

ITS2-PCR. The ITS2 region was amplified with primers ITS86 $\left(5^{\prime}-\right.$ GTGAATCATCGAATCTTTGAAC-HEX-3') (fluorescently labelled) and ITS4 (5'-TCCTCCGCTTATTGATATGC- $\left.3^{\prime}\right)$. The ITS86 primer was used as a mixture of $20 \%$ fluorescently labelled and $80 \%$ nonfluorescently labelled primer in order to avoid out-of-range peak heights. The PCR mix contained $25 \mu \mathrm{l}$ mastermix (Qiagen), $0.5 \mu \mathrm{M}$ each primer, $5 \mu \mathrm{l}$ DNA extract and sterile distilled water to a final volume of $50 \mu \mathrm{l}$. Thermal cycling was carried out according to the following protocol: $5 \mathrm{~min}$ at $94{ }^{\circ} \mathrm{C} ; 30$ cycles of $1 \mathrm{~min}$ at $94{ }^{\circ} \mathrm{C}, 1 \mathrm{~min}$ at $55{ }^{\circ} \mathrm{C}$ and $1 \mathrm{~min}$ at $72{ }^{\circ} \mathrm{C}$; a final extension of $7 \mathrm{~min}$ at $72{ }^{\circ} \mathrm{C}$; and cooling at $4{ }^{\circ} \mathrm{C}$.

BstUI restriction digestion of the amplified ITS2 region. Two microlitres of the amplification product was transferred to a new

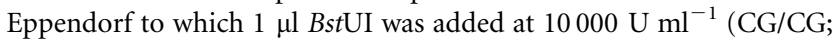
New England BioLabs), $2 \mu \mathrm{l} B s t \mathrm{UI}$ restriction buffer and $15 \mu \mathrm{l}$ distilled water. The mixture was incubated for $2 \mathrm{~h}$ at $60{ }^{\circ} \mathrm{C}$.

Following restriction, the $20 \mu \mathrm{l}$ DNA restriction digestion mixture was purified by precipitation by adding $2 \mu \mathrm{l} 3 \mathrm{M}$ sodium acetate, $50 \mu \mathrm{l} 95 \%$ ethanol and $0.2 \mu \mathrm{l} 20 \mathrm{mg}$ dextran blue $\mathrm{ml}^{-1}$, followed by centrifugation at $10000 \mathrm{~g}$ for $30 \mathrm{~min}$. The pellet was washed with $250 \mu \mathrm{l} 70 \%$ ethanol and air dried.

Fragment length determination. Formamide $(11.5 \mu \mathrm{l}), 1 \mu \mathrm{l}$ nondigested ITS2 amplicon, $0.3 \mu$ HD400 marker (Applied Biosystems) and $0.1 \mu \mathrm{l}$ ROX500 marker (Applied Biosystems) were added to the ethanol precipitate of the digest. This mixture was electrophoresed on an ABI 310 capillary electrophoresis system (Applied Biosystems), as described previously (De Baere et al., 2002).

RFLP analysis. Fragment length determination analysis was carried out using GENESCAN analysis software (Applied Biosystems) and final fragment length analysis and comparison of the obtained fingerprints was carried out using the differential base-pair algorithm of the Basehopper software, as developed previously (Baele et al., 2001). Briefly, a set of fingerprints of reference strains, designated library entries, was used to make a library. Each library entry was composed of two numbers, whereby the first number represented the $5^{\prime}$ terminal restriction fragment in base pairs and the second number represented the fragment length in base pairs of the non-digested ITS2 for that strain. Fingerprints of unknown strains, also each composed of two numbers, representing the fragment lengths in base pairs, were then compared with the library entries.

ITS2 gene sequence determination. Further discrimination and additional data on identity were obtained by ITS2 gene sequencing. The ITS2 sequence obtained was compared with all sequences submitted to GenBank using the BLAST software at NCBI. Supplementary Table S1 presents the sequence data obtained for a subset of strains during this study.

\section{RESULTS AND DISCUSSION}

ITS2-RFLP patterns consisted basically of two numbers, representing the terminal $5^{\prime} B s t \mathrm{UI}$ restriction fragment length and the full-length ITS2 fragment length in base pairs. The consensus fingerprints constructed for each species are listed in Table 1. When not all strains of a species had the same ITS2-RFLP pattern, the species was subdivided into groups, corresponding to the different ITS2-RFLP patterns observed.

In total, the following 12 species could be identified unambiguously on the basis of ITS2-RFLP analysis, because of the presence of a unique pattern, not observed in any other species included in this study: Arthroderma cajetani (one strain), Arthroderma grubyi (three), Arthroderma gypseum (three), Arthroderma incurvatum (three), Arthroderma racemosum (one), Arthroderma simii (two), Arthroderma uncinatum (three), Chrysosporium georgiae (one), Chrysosporium merdarium (one), Epidermophyton floccosum (two), Microsporum praecox (four) and Trichophyton balcaneum (one).

In addition, two other species, Arthroderma obtusum and Microsporum audouinii, could be identified unambiguously, although some genotypic diversity was present within these species.

The three A. obtusum (anamorph Microsporum nanum) strains studied could be divided into two ITS2-RFLP subgroups. Strain CBS 314.54 had a fingerprint of 137.8 and $327.3 \mathrm{bp}$. The second group consisted of CBS 727.88 and CBS 728.88, with an ITS2-RFLP fingerprint composed of 135.8 and 324.4 bp fragments. Both A. obtusum ITS2RFLP groups presented unique patterns not observed for any other species tested. CBS 314.54 coincided with the original Mexican type strain of the anamorph M. nanum, and CBS 727.88 and CBS 728.88 represented isolates from Canada and France, respectively. It is possible that this species, which is associated with both wild and domestic pigs, has some worldwide diversity. All world isolates can interbreed as far as is known. Thus, in total, 8 of the 15 Arthroderma species could be differentiated from each other and from other genera.

In addition, the two $M$. audouinii strains revealed two different fingerprints, but as both were not encountered for 
Table 1. Species and strains used in this study, with the ITS2-RFLP pattern represented by two fragments, i.e. the 5' terminal BstUI restriction fragment and the full-length ITS2 fragment

Underlined strains indicate sequences that were determined in this study (detailed sequence information is available in Supplementary Table S1, available in JMM Online).

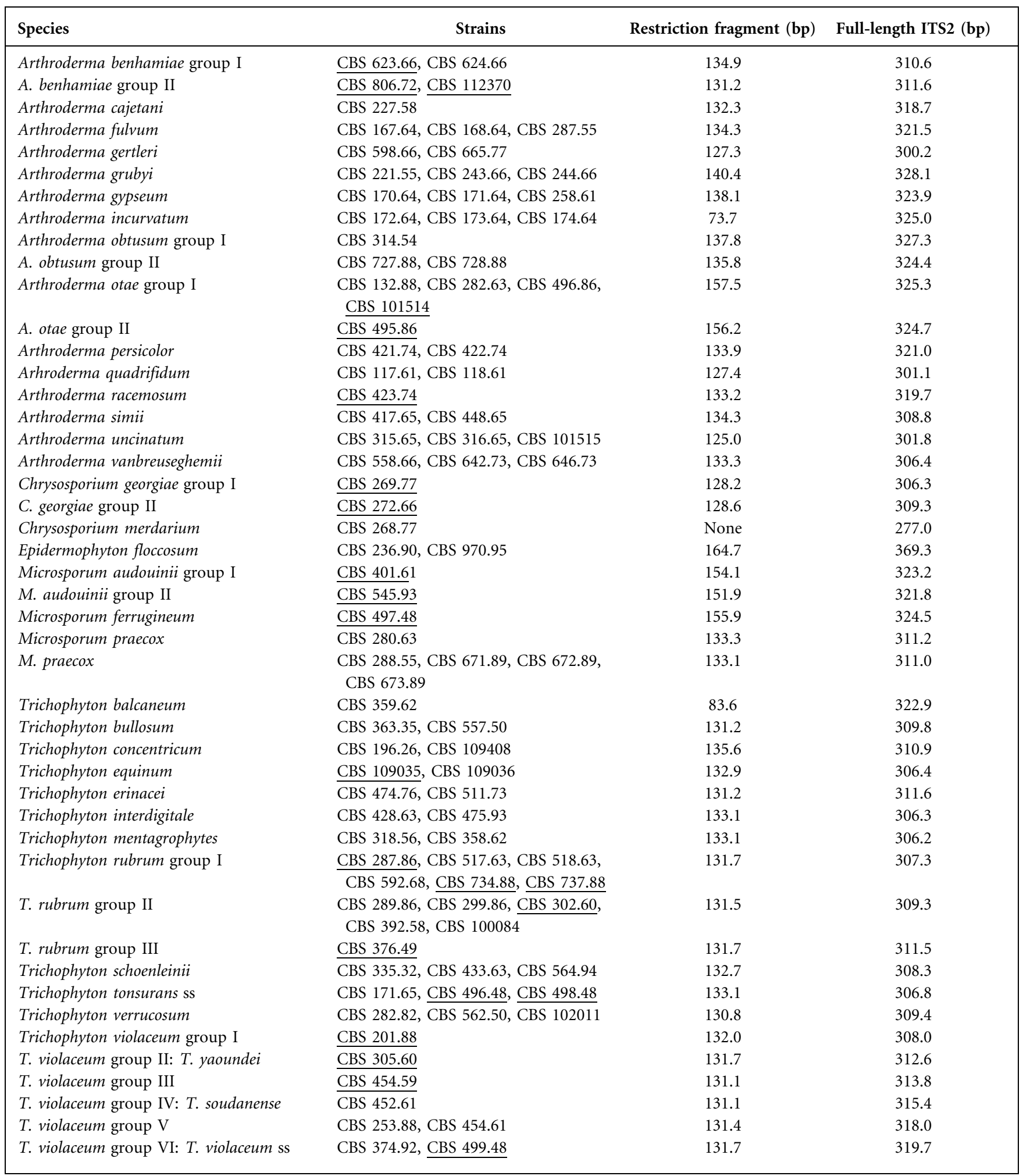


any other species, identification of this species remains possible as well. We cannot explain why the two fingerprints differed, as the sequences of both strains were identical.

The other species analysed here yielded some identification problems.

The four Arthroderma benhamiae strains studied here could be separated into two groups based on their ITS2-RFLP pattern. A. benhamiae consists of a complex set of partially interbreeding populations and it is not clear how many different ITS2-RFLP groups there may be worldwide (Summerbell et al., 1999). The standard mating type tester isolates CBS 623.66 and CBS 624.66, designated A. benhamiae ITS2 group I, with ITS2-RFLP pattern 134.9, 310.6, have a North American origin $[A$. benhamiae (-) mating type]. The ITS2-RFLP pattern of the A. benhamiae ITS2 group I strains was indistinguishable from that of the Trichophyton concentricum strains.

The A. benhamiae ITS2 group I pattern did not show similarity to that of the European isolates 806.72 and CBS 112370, designated A. benhamiae ITS2 group II [A. benhamiae $(+)$ mating type], which were both sequenced in this study and confirmed as genuine A. benhamiae. These isolates are, however, part of the same interbreeding pool, the 'Americano-European' A. benhamiae. The other interbreeding pool (marginally capable of crossing with the Americano-European pool) is the African A. benhamiae, which also includes all isolates from hedgehogs in Africa, Europe and New Zealand, but which was not included in this study. The European isolate CBS 806.72 probably belongs to a genetic subtype of this second interbreeding pool that is specific to guinea pigs (except when it crosses over to infect their human handlers). The ITS2-RFLP pattern of the A. benhamiae group II was indistinguishable from that of Trichophyton erinacei, which is in accordance with recent taxonomic insights classifying $A$. benhamiae as the teleomorph species of $T$. erinacei (also from hedgehogs) (Nenoff et al., 2007). The pattern was also indistinguishable from the ITS2-RFLP fingerprints of the Trichophyton rubrum ITS2 group III and Trichophyton violaceum group II.

The three Arthroderma fulvum strains had an identical ITS2-RFLP pattern, indistinguishable from that observed for the two Arthroderma persicolor strains. The clinical importance of both species is comparable and thus this similarity would pose no diagnostic difficulties. Furthermore, in silico analysis predicted that both species could be differentiated by digestion with, for example, $M b o$ (/GATC), for which there are two restriction sites in the ITS2 sequence of A. fulvum (positions 123 and 278), but only one restriction site in the ITS2 sequence of $A$. persicolor (position 278).

The two Arthroderma gertleri strains had an ITS2-RFLP pattern closely related to that of the two Arthroderma quadrifidum strains, such that discrimination between both species is cumbersome, because the difference is at the limit of the $1 \mathrm{bp}$ resolution of the ABI310 electrophoresis apparatus.

As mentioned above, the three A. grubyi strains had an identical pattern that was not observed for any other species. However, this species includes two different types of strain. One is a group of sexually reproducing soil fungi (anamorph Microsporum vanbreuseghemii, isolates CBS 243.66 and CBS 244.66) and the other is a morphologically different asexual lineage specific to chickens and related gallinaceous fowl, occasionally infecting a human handler or farm dog (Microsporum gallinae strain CBS 221.55). The ITS2-RFLP analysis did not separate these two very closely related entities. Both are infectious in humans, although neither persists in human populations. Their clinical significance is therefore identical, except that control of a bird outbreak might help in the control of specific $M$. gallinae infections.

Four of the five Arthroderma otae strains (CBS 101514, CBS 132.88, CBS 282.63 and CBS 496.86) had an identical pattern $(157.5,325.3)$, which was not observed for any other species.

A. otae is a complex containing a sexual species native to the cat family, with its anamorphic form Microsporum canis, and two non-interbreeding, morphologically and biomedically different, human-associated, asexual radiate lineages, the African $M$. audouinii and the Asian Microsporum ferrugineum. The species $M$. canis, most frequently isolated from cats (and dogs) and which also regularly infects humans, consists almost entirely of the (-) mating type, whereas the $(+)$ mating type has rarely been found and only in Japan.

A. otae group II strain CBS 495.86 is the (+) mating type tester strain and had a pattern indistinguishable from that of strain CBS 497.48, included in this study as $M$. ferrugineum. The two human lineages $M$. audouinii and $M$. ferrugineum have both been shown to be more closely related to the $(+)$ mating type tester than to the $(-)$ mating type M. canis isolates and presumably evolved from the $(+)$ mating type isolates. Therefore, the inability to differentiate $A$. otae CBS 495.86 (+) from M. ferrugineum CBS 497.48 and the grouping of A. otae/M. canis isolates CBS 101514, CBS 132.88 and CBS 282.63 with the (-) mating type tester CBS 496.86 are in accordance with current taxonomical insights. Sequence determination of CBS 495.86 and CBS 101514 confirmed their identity to $A$. otae (GenBank accession no. AJ252339) (A. otae group II in this study) and $M$. canis/Microsporum distortum (GenBank accession no. AJ252328) (A. otae group I in this study), respectively.

The two A. quadrifidum strains had a pattern that could easily be distinguished from that of the three A. uncinatum strains included. Both species are considered as contaminants in the clinical laboratory. As mentioned above, the ITS2-RFLP pattern of A. quadrifidum was, however, closely 
related to that of $A$. gertleri, leading to the inability to discriminate between the two species.

The three Arthroderma vanbreuseghemii strains CBS 558.66, CBS 642.73 and CBS 646.73 had an ITS2-RFLP pattern that was indistinguishable from that of the strains of the species Trichophyton equinum, Trichophyton mentagrophytes and Trichophyton tonsurans. The A. vanbreuseghemii pattern was also not distinguishable from that of its anamorph, i.e. T. interdigitale.

T. tonsurans is known to be a very close relative of $A$. vanbreuseghemii, whilst $T$. mentagrophytes is relatively close, but no closer than some other species found to be different by ITS2-RFLP analysis, such as T. balcaneum and A. simii. The $A$. vanbreuseghemii/T. tonsurans clade contains four medically distinct entities: (i) A. vanbreuseghemii itself (CBS 642.73, CBS 646.73), a sexual species growing on rodents and occasionally infecting humans; (ii) Trichophyton interdigitale (CBS 428.63, CBS 475.93), a human-associated asexual lineage growing mainly on adult feet and very closely related to, and descended from, the (-) mating type of $A$. vanbreuseghemii; (iii) T. tonsurans sensu stricto (CBS 171.65, CBS 498.48), a purely human-infecting asexual species mainly causing scalp ringworm in children; and (iv) T. equinum (CBS 109035, CBS 109036), an asexual dermatophyte infecting horses and occasionally their human contacts.

The two strains included as C. georgiae, an anamorph of Arthroderma ciferrii, were found to be different from each other, with both strains having a unique pattern. The genotypic difference between both C. georgiae ITS2 groups was reflected by the fact that strain CBS 272.66 produced a red pigment, whereas strain CBS 269.77 produced a white/ yellow pigment. Sequencing indicated that CBS 272.66 is identical to C. georgiae (GenBank accession no. AJ007844), whereas the sequence of CBS 269.77 had highest similarity $(89 \%)$ to GenBank accession no. AB219227, which was deposited as Chrysosporium carmichaelii. It is likely that CBS 269.77 belongs to a species for which the ITS2 sequence is not yet included in GenBank. The obtained sequence was submitted to GenBank as FM212440.

The C. merdarium strain was the only strain in this study that was not digested during the restriction reaction using $B s t \mathrm{UI}$. Because of this, the resulting unique ITS2-RFLP fingerprint consisted of only one fragment.

The single $M$. ferrugineum strain studied could not be differentiated by ITS2-RFLP analysis from the A. otae ITS2 group II strain CBS 495.86, as discussed above.

As mentioned above, the four $M$. praecox strains had an identical pattern not observed for any other species. In addition, strain CBS 280.63, received as M. audouinii, was identified as M. praecox on the basis of ITS2-RFLP analysis. This finding was confirmed by ITS2 sequencing, yielding $100 \%$ similarity to $M$. praecox.

The greatest number of problems was encountered within the genus Trichophyton.
The two Trichophyton bullosum strains had an ITS2-RFLP pattern that was indistinguishable from that of T. rubrum ITS2-RFLP genotype II and from T. verrucosum. The two T. concentricum strains had an ITS2-RFLP pattern that was indistinguishable from that of the A. benhamiae group I (of the North American A. benhamiae mating type). This human-associated species, causing a dramatic chronic disease (tinea imbricata) but only in certain genetically predisposed people of Austronesian ancestry, is considered a sister species of $A$. benhamiae, in agreement with the close resemblance between their ITS2-RFLP patterns.

The ITS2-RFLP pattern of the two T. equinum strains was indistinguishable from that of T. tonsurans, and was also very similar to those of the other species in the $A$. vanbreuseghemii clade, as discussed above. The two $T$. erinacei strains, as mentioned above, were not distinguishable by ITS2-RFLP from A. benhamiae group II (the European A. benhamiae group), which is the teleomorph species, and from $T$. rubrum group III. The two $T$. interdigitale strains had an ITS2-RFLP pattern identical to that of A. vanbreuseghemii, the teleomorph.

The two T. mentagrophytes (teleomorph A. vanbreuseghemii) strains could not be differentiated from $T$. interdigitale, T. equinum and T. tonsurans, as discussed in the section on A. vanbreuseghemii.

The $12 T$. rubrum strains fell into three ITS2 groups. T. rubrum ITS2 group I consisted of the six strains CBS 287.86, CBS 517.63, CBS 518.63, CBS 592.68, CBS 734.88 and CBS 737.88, which had an ITS2-RFLP fingerprint of 131.7 and 307.3 bp. Strains CBS 287.86 and CBS 592.68 are Afro-Asiatic. Strains CBS 287.86, CBS 734.88 and CBS 737.88 were sequenced and all contained a six-TA repeat at position 162 of the ITS2 region. T. rubrum ITS2 group I could not be differentiated from Trichophyton violaceum ITS2 group I and Trichophyton schoenleinii.

T. rubrum ITS2 group II consisted of five strains, CBS 100084, CBS 302.60, CBS 392.58 (neotype), CBS 289.86 and CBS 299.86, and had a fingerprint that differed from the T. rubrum ITS2 group I strains by only 2 bp over the total length of the ITS2. Sequencing of strain CBS 100084 revealed seven TA repeats at position 162 of the ITS2 region, explaining the $2 \mathrm{bp}$ difference with $T$. rubrum ITS2 group I strains, carrying only six TA repeats. T. rubrum ITS2 group II strains could not be differentiated from $T$. bullosum and T. verrucosum.

T. rubrum ITS2 group III consisted of one strain, CBS 376.49 , the type strain of Trichophyton rodhaini, an invalidly described species that is currently considered to be part of the T. rubrum complex (Gräser et al., 2000). The ITS2-RFLP region of this strain contained eight TA repeats and this group could not be distinguished from $T$. erinacei and from A. benhamiae ITS2 group II, i.e. the teleomorph species of T. erinacei.

The three T. schoenleinii strains had an ITS2-RFLP pattern that was closely related to the fingerprint of $T$. violaceum 
ITS2-RFLP group I. T. schoenleinii, a now-rare agent of the most severe form of human dermatophytosis, was easily distinguishable from the close relative T. mentagrophytes, the mouse dermatophyte, which also infects humans coming into contact with mouse fomites.

The three strains of $T$. tonsurans sensu stricto had an identical ITS2-RFLP pattern, as discussed above for $A$. vanbreuseghemii. Recently, three ITS haplotypes have been recognized within the $T$. tonsurans/T. equinum group (Summerbell et al., 2007). One haplotype has base C at position 18 and a series of nine adenine bases starting at position 201, and corresponds to T. tonsurans sensu stricto. Another, with $\mathrm{T}$ at position 18 and nine adenine bases starting at position 201, corresponds to T. equinum and T. equinum var. autotrophicum. The third, with $\mathrm{T}$ at position 18 and 11 adenine bases starting at position 201, corresponds to an organism that has been referred to as ' $T$. tonsurans DNA type 2'. ITS2-RFLP may be able to differentiate between the two first versus the third haplotype, but apparently none of the strains in this study belonged to the third haplotype.

The pattern of the three strains of $T$. verrucosum was indistinguishable from that of T. bullosum and T. rubrum ITS2 group II.

There was a high degree of variability in ITS2-RFLP patterns among the eight $T$. violaceum isolates studied, resulting in six different ITS2 groups (Table 1). However, all had a restriction fragment of $131.5 \mathrm{bp}$ (mean value, SD 0.35 ) in common. The same restriction fragment length was also obtained for T. rubrum isolates.

The following $T$. violaceum strains were studied: CBS 201.88 (sequence identical to GenBank accession nos AF170473 and AF170474, listed as Trichophyton soudanense, and to AF170463 and AF170464, listed as Trichophyton megninii), CBS 305.60 (central African Trichophyton yaoundei strain, sequence $99 \%$ similar to GenBank accession no. EU181450, listed as T. violaceum), CBS 454.59 (sequence identical to GenBank accession no. EU181450, listed as T. violaceum), CBS 452.61 (subSaharan African $T$. soudanense, sequence identical to GenBank accession no. EU200402, listed as T. soudanense), CBS 253.88 and CBS 454.61 (sequence $99 \%$ similar to GenBank accession no. AJ270796, listed as T. violaceum), CBS 374.92 (African/Middle Eastern T. violaceum sensu stricto, sequence identical to GenBank accession no. AJ270796, listed as T. violaceum) and CBS 494.48 (sequence identical to GenBank accession no. AJ270796, listed as T. violaceum, formerly listed as Trichophyton glabrum). The different groups all had the same sequence, only differing in the number of TA repeats.

T. violaceum has traditionally been divided into four species, namely T. glabrum, T. yaoundei, T. soudanense and $T$. violaceum, which were then grouped together after a sequencing study (Gräser et al., 2000). These entities are all closely related to $T$. rubrum, although they cause a different spectrum of diseases; the $T$. violaceum sensu lato group (including T. soudanense and T. yaoundei) all cause scalp ringworm, mainly in children, whilst $T$. rubrum causes lower-body and especially foot dermatophytosis, almost always in adults. The close resemblance of the $T$. violaceum ITS2-RFLP patterns to those of T. rubrum was therefore not unexpected. More generally, the larger clade inclusive of T. rubrum and T. violaceum is ultimately an offshoot of A. benhamiae, so the resemblance between A. benhamiae and T. violaceum subsp. yaoundei was also not unexpected.

In summary, ITS2-RFLP analysis, although limited for differentiation of Trichophyton species, was found to be an informative molecular approach, the results of which are in agreement with modern taxonomic insights based on clinical, phenotypic and molecular data.

Although this study dealt with the applicability of the ITS2 region to identifying dermatophyte fungi and focused on the application of a DNA fingerprinting approach, sequencing of the ITS2 region or - even better - both ITS1 and ITS2 regions (Gemmer et al., 2002), provides the most relevant information, which can also be exchanged easily between laboratories. However, the approach outlined here might be used for quick screening of large collections of unknown isolates prior to more laborious sequencing of those isolates that cannot be allocated unambiguously to one of the described taxa on the basis of ITS2-RFLP analysis.

\section{ACKNOWLEDGEMENTS}

T.D. B. is indebted to the FWO (Fonds voor Wetenschappelijk Onderzoek - Vlaanderen) for a postdoctoral fellowship.

\section{REFERENCES}

Arabatzis, M., Xylouri, E., Frangiadaki, I., Tzimogianni, A., Milioni, A., Arsenis, G. \& Velegraki, A. (2006). Rapid detection of Arthroderma vanbreuseghemii in rabbit skin specimens by PCR-RFLP. Vet Dermatol 17, 322-326.

Arabatzis, M., Bruijnesteijn van Coppenraet, L. E., Kuijper, E. J., de Hoog, G. S., Lavrijsen, A. P., Templeton, K., van der Raaij-Helmer, E. M., Velegraki, A., Gräser, Y. \& Summerbell, R. C. (2007). Diagnosis of common dermatophyte infections by a novel multiplex real-time polymerase chain reaction detection/identification scheme. $\mathrm{Br} J$ Dermatol 157, 681-689.

Baele, M., Storms, V., Haesebrouck, F., Devriese, L. A., Gillis, M., Verschraegen, G., de Baere, T. \& Vaneechoutte, M. (2001). Application and evaluation of the interlaboratory reproducibility of tRNA intergenic length polymorphism analysis (tDNA-PCR) for identification of Streptococcus species. J Clin Microbiol 39, 1436-1442.

De Baere, T., Claeys, G., Swinne, D., Verschraegen, G., Muylaert, A., Massonet, C. \& Vaneechoutte, M. (2002). Identification of cultured isolates of clinically important yeast species using fluorescent fragment length analysis of the amplified internally transcribed rRNA spacer 2 region (ITS2). BMC Microbiol 2, 21.

De Baere, T., Van Keerberghen, A., Van Hauwe, P., De Beenhouwer, H., Boel, A., Verschraegen, G., Claeys, G. \& Vaneechoutte, M. (2005). An interlaboratory comparison of ITS2-PCR for the identification of yeasts, 
using the ABI Prism 310 and CEQ8000 capillary electrophoresis systems. BMC Microbiol 5, 14.

Erhard, M., Hipler, U. C., Burmester, A., Brakhage, A. A. \& Wostemeyer, J. (2008). Identification of dermatophyte species causing onychomycosis and tinea pedis by MALDI-TOF mass spectrometry. Exp Dermatol 17, 356-361.

Gemmer, C. M., DeAngelis, Y. M., Theelen, B., Boekhout, T. \& Dawson, T. L., Jr (2002). Fast, noninvasive method for molecular detection and differentiation of Malassezia yeast species on human skin and application of the method to dandruff microbiology. J Clin Microbiol 40, 3350-3357.

Gräser, Y., El Fari, M., Vilgalys, R., Kuijpers, A. F., De Hoog, G. S., Presber, W. \& Tietz, H. (1999). Phylogeny and taxonomy of the family Arthrodermataceae (dermatophytes) using sequence analysis of the ribosomal ITS region. Med Mycol 37, 105-114.

Gräser, Y., Kuijpers, A. F., Presber, W. \& de Hoog, G. S. (2000). Molecular taxonomy of the Trichophyton rubrum complex. J Clin Microbiol 38, 3329-3336.
Nenoff, P., Herrmann, J. \& Gräser, Y. (2007). Trichophyton mentagrophytes sive interdigitale? A dermatophyte in the course of time. J Dtsch Dermatol Ges 5, 198-202.

O’Donnell, K., Cigelnik, E., Weber, N. S. \& Trappe, J. M. (1997). Phylogenetic relationships among ascomycetous truffles and the true and false morels inferred from $18 \mathrm{~S}$ and $28 \mathrm{~S}$ ribosomal DNA sequence analysis. Mycologia 89, 48-65.

Summerbell, R. C., Haugland, R. A., Li, A. \& Gupta, A. K. (1999). rRNA gene internal transcribed spacer 1 and 2 sequences of asexual, anthropophilic dermatophytes related to Trichophyton rubrum. J Clin Microbiol 37, 4005-4011.

Summerbell, R. C., Moore, M. K., Starink-Willemse, M. \& Van Iperen, A. (2007). ITS barcodes for Trichophyton tonsurans and T. equinum. Med Mycol 45, 193-200.

Turenne, C. Y., Sanche, S. E., Hoban, D. J., Karlowsky, J. A. \& Kabani, A. M. (1999). Rapid identification of fungi by using the ITS2 genetic region and an automated fluorescent capillary electrophoresis system. J Clin Microbiol 37, 1846-1851. 